\title{
Interdisciplinary Ensembles: inglés y creatividad multidisciplinaria artística en la Facultad de Música de la UNAM.
}

\author{
Interdisciplinary Ensembles: English and Artistic Multidisciplinary Creativity at the Faculty of \\ Music, UNAM \\ Thelma J. G. Pérez-Vilchis ${ }^{a}$
}

\begin{abstract}
:
Creation of the Interdisciplinary Ensembles workshop at the Faculty of Music (UNAM) as an ad hoc Elective Subject to satisfy the English language practice needs of undergraduate students five academic semesters ago is presented. The workshop is mainly focused on students from instrumentalist careers. There, English language is practiced in the musical field (particularly) and in the artistic field (in general) through exploration of the relationships among music, use of words (narration, poetry, dialogue, etc.), and the body (in movement, dancing, acting, etc.) from emotional and artistic perspectives.
\end{abstract}

\section{Keywords:}

English, artistic multidiscipline, creativity, music, literature, body

\section{Resumen:}

Se presenta la creación del taller Interdisciplinary Ensembles de la Facultad de Música de la Universidad Nacional Autónoma de México (UNAM) como Materia Optativa ad hoc para satisfacer las necesidades de práctica del idioma inglés de los estudiantes de licenciatura hace cinco semestres académicos. El taller está dirigido, principalmente, a estudiantes de las carreras de instrumentista a nivel licenciatura. En el mismo se practica el idioma inglés en las áreas musical (en especial) y artística (en general) por medio de la exploración de las relaciones entre música, el uso de la palabra (narración, poesía, diálogo, etc.) y el cuerpo (en movimiento, bailando, actuando, etc.) desde las perspectivas emocional y artística.

\section{Palabras Clave:}

inglés, multidisciplina artística, creatividad, música, literatura, cuerpo

\section{ANTECEDENTES}

En el año 2017, la Facultad de Música de la UNAM se interesó por ofrecer a sus estudiantes de licenciatura una alternativa de materia optativa para practicar el idioma inglés en su área profesional. Se contaba ya con la materia seriada de cuatro semestres de enseñanza del idioma, pero no con un espacio para la práctica del mismo en el área musical (de manera específica) y artística (de manera general). Durante charlas de planeación, se partió de la posibilidad de impartir una materia teórica curricular en inglés. Se descartó la idea pues siendo la materia propuesta fundamental en la formación musical básica de los estudiantes, era arriesgado exponer a los alumnos que apenas adquirían los conocimientos de la misma en su lengua materna al desconocimiento agregado de una lengua extranjera. Esto hubiera sido un despropósito, al presentar complicaciones en el proceso formativo en lugar de enriquecerlo.

Se propuso, pues, la asignatura Interdisciplinary Ensembles. La descripción, de acuerdo con el Programa de Asignatura presentado a la dirección de la FaM y aprobado por la misma es el siguiente:

This workshop is based on the work of the students in the Chamber Music subjects; it allows the students who have already studied English to practice their

Corresponding author, Universidad Nacional Autónoma de México (UNAM), https://orcid.org/0000-0003-1709-7155, Email: jaet.garibaldi@yahoo.com 
knowledge of the language in projects that become rehearsals for the professional practice; it reinforces an attitude of openness towards other artistic languages in the students and diversifies their expressive resources.

The interpreter plays and occupies the stage with his/her body. Therefore, s/he must be conscious of it during musical performance. The performing musician may use words as a vehicle to take music closer to the audience in an attractive and accessible manner. That is why it is important for the students to identify parallelisms between oral and musical language and be capable of choosing and/or creating brief texts that are pertinent and relevant to the music they want to play. The body of the instrumentalist may also function as an image to enrich musical performance. Hence the usefulness of being capable to design body movements consistent with the music and in accordance with the students' physical skills and capabilities. (Garibaldi, 2017)

Con objetivo general:

Participants will enrich their musical performance on the stage with literature and body movement elements that are pertinent and relevant to the works they play, whether choosing them or creating them, contextualizing them, and justifying them orderly. They will be conscious of their own body on stage and will convey thoughts and emotions through music, words and movement. Upon completion of the course, the students will make a public presentation where they will also explain orally their work process in English. (Garibaldi, 2017)

El acercamiento a la creatividad en la multidisciplina artística permite experimentar con diversos medios de indagación en las propias emociones; diversifica y enriquece los recursos de construcción de la propia identidad; y promueve una actitud de apertura hacia diversas formas de expresión, tanto artísticas como humanas.

Entiéndase en este trabajo "multidisciplina artística" como el acercamiento formal a varias disciplinas artísticas. Con "formal" se hace referencia a una formación seria, no necesariamente académica, pero sí estructurada y profunda, bien fundamentada en los principios básicos de cada área. La autora se basa en su propia experiencia profesional como creadora multidisciplinaria (Lic. en Letras Inglesas, Lic. en Flauta Transversa, compositora, escritora, bailarina); perito traductora; y profesora de la materia optativa de Interdisciplinary Ensembles.

\section{PLANTEAMIENTO}

De acuerdo con las necesidades que se solicitaba cubrir, el taller es un espacio de práctica del idioma inglés que complementa los posibles estudios musicales de los participantes tanto en la FaM como fuera de ella, así como su futura actividad profesional. En este tenor, no hay restricción en relación con el nivel de lengua previo. Han llegado igualmente estudiantes que ya han tenido experiencias reales con el inglés en el extranjero, sea por medio de clases magistrales o cursos de mayor duración, así como estudiantes con gran dificultad para expresarse y comprender el idioma, que han enfrentado la necesidad de comprenderlo en clases y cursos impartidos en inglés en nuestro país por músicos de talla internacional (sea su lengua materna o no).

A fin de trabajar con grupos heterogéneos en cuanto a los niveles musical y de lengua inglesa, la autora abreva de su experiencia formativa en flauta transversa en la FaM bajo la tutela de Miguel Ángel Villanueva, con quien trabajó la metodología de clase grupal. Cobo considera que "l'apprentissage collaboratif vise l'interdépendance sociale et cognitive entre les élèves afin de stimuler l'interaction critique", (p. 32) por lo que los participantes se ven beneficiados por los comentarios de sus compañeros en tanto que la crítica constituye comentarios constructivos a las creaciones de los demás. Por su parte, Crousier indica que "les élèves les plus avancés peuvent faire part de leur expérience aux plus jeunes, tandis que les conseils du professeur aux plus jeunes servent de rappel pour les plus avancés". (p. 84) Si bien Crousier se refiere a la clase instrumental grupal, esto aplica tanto en el área musical como en la de lengua, pues tener cerca la referencia de alguien con mayor nivel puede ser motivante para los alumnos menos avanzados (no necesariamente los de menor edad).

Como el nombre del taller indica, las dinámicas propuestas en los ejercicios específicos al inicio del curso así como el trabajo basado en las obras de los participantes, se relacionan directamente con la práctica profesional de los estudiantes en tanto que el trabajo de ensamble es inherente a las profesiones musicales, sea como intérpretes, alumnos, profesores o compositores con sus intérpretes, por nombrar algunas. Durante las sesiones, la profesora señala cómo lo trabajado en el taller se podría trasladar a experiencias de la vida profesional.

Se considera que mientras los participantes se atrevan a participar activamente con sus compañeros, buscarán la manera de darse a entender, entre otros, buscando los recursos específicos de lengua como son vocabulario musical y general (e.g.: por medio del uso del celular y la red de Internet de la $\mathrm{FaM}$ ): "la notion première du bon fonctionnement d'un groupe : la communication. Celle-ci sera bien sûr musicale, mais aussi verbale et non verbale." (Crousier, p. 85) Se ha observado que la introversión y la inseguridad son factores más importantes que el nivel de dominio del inglés para fines de interacción entre los participantes, desarrollo de sus ideas creativas e interpretación de las piezas de sus compañeros y, por ende, para el progreso en su manejo del idioma inglés. Por ello: "il est bon d'aider les plus timides, de tempérer les impulsifs ; chacun doit pouvoir s'exprimer à son rythme et 
selon ses compétences. » (Biget, p. 25) Más que moderar los impulsos de los participantes más arrojados, como indica Biget, dada la naturaleza de la actividad musical, la profesora invita a los participantes a canalizarlos por medio de la creatividad y la interpretación conscientes.

La autora ha percibido, también, que la mayor barrera para comenzar un trabajo creativo que incluya el uso del inglés consiste en los conceptos preconcebidos de los estudiantes en torno a lo válido -o no- que es expresar sus propias opiniones e ideas. De ahí la decisión de presentar ejercicios específicos que ejemplifiquen diferentes formas creativas de usar recursos de diversas disciplinas artísticas. Se espera, por medio del ejemplo, animar a los participantes a explorar sus intereses y sus propias formas de expresión, a fin de encontrar una motivación profunda que los motive de manera natural a allegarse voluntariamente más recursos de aprendizaje del inglés, sea de manera autodidacta o por medio de clases formales de idioma (en o fuera de la FaM). "Cette méthode qui consiste à échanger, discuter pour construire, cette maïeutique socratique (l'art de faire découvrir à son interlocuteur, par une série de questions, les vérités qu'il porte en lui) est difficile à utiliser. Le professeur, autant que les élèves, doit en faire l'apprentissage. » (Biget, p. 25) En el caso de este taller, las "verdades" que refiere Biget son los conocimientos y/o las carencias de los propios participantes, lo que se construye son las piezas creativas y la mayéutica se da en el momento en que los estudiantes buscan darse a entender con sus compañeros. Una vez que los participantes plantean su línea de trabajo, la profesora señala continuamente los recursos necesarios para llevar a cabo las ideas propuestas y si ella observa que los estudiantes ya cuentan con ellos o no. Se realizan entonces ajustes entre lo que pretenden y lo que pueden realizar, hasta llegar al punto en que apliquen los conocimientos que ya tienen y, en la medida de lo posible en términos de capacidad y medios, busquen profundizar su conocimiento de la lengua en cuanto a vocabulario y gramática. Maria Montessori refiere que "discipline must come through liberty." (p. 113) La autora considera que la disciplina y el interés por aprender se basan en la motivación interna de cada estudiante y que, efectivamente, sólo se puede contactar con éstos cuando cada persona tiene la libertad de buscar en sí lo que realmente siente, piensa y se le permite expresarlo.

Si bien el taller es, por su naturaleza, netamente experimental, en relación con el estudio del idioma inglés es importante señalar que, independientemente del nivel objetivo de lengua que tengan los alumnos, lo más importante es lograr que los mismos encuentren utilidad práctica y expresiva en el uso del inglés a fin de que continúen o emprendan sus estudios de la lengua con entusiasmo y autodisciplina, teniendo claro para qué requieren los conocimientos de la misma.
Communiquer, c'est vouloir transmettre aux autres, à l'aide d'un langage commun et compréhensible, des idées, des désirs, des émotions, dans le but d'installer une relation constructive. Ici, le rôle de l'enseignant sera de faire prendre conscience à chaque élève des difficultés inhérentes à tout système de communication. (Crousier, p. 86)

En este caso, los lenguajes comunes son la música y el inglés, pues los demás elementos de las piezas quedan a elección de cada quien. Se espera que los estudiantes reconozcan la aplicación del estudio cotidiano de la técnica de su instrumento, así como de las materias teóricas, que suele resultarles tedioso y, en muchos casos, sinsentido. Además, se pretende que se hagan conscientes de que ya tienen otros conocimientos fuera del ámbito musical de los que pueden echar mano para enriquecer sus experiencias interpretativa y creativa. Sea que los participantes ya hayan estudiado formalmente (no por fuerza en una institución) o no inglés, el idioma puede ser uno de tales elementos que les permita ampliar su uso consciente de la palabra en todas sus formas.

A la fecha, los alumnos que han participado en el taller han llegado sin experiencia creativa previa en inglés. Al concluir el semestre, han elaborado un partitura-guion en la cual se basaron ellos mismos y sus compañeros para interpretar sus piezas. El esfuerzo involucrado en poner por escrito sus ideas implica, en sí mismo, un progreso de los estudiantes en cuanto a su interés en el idioma.

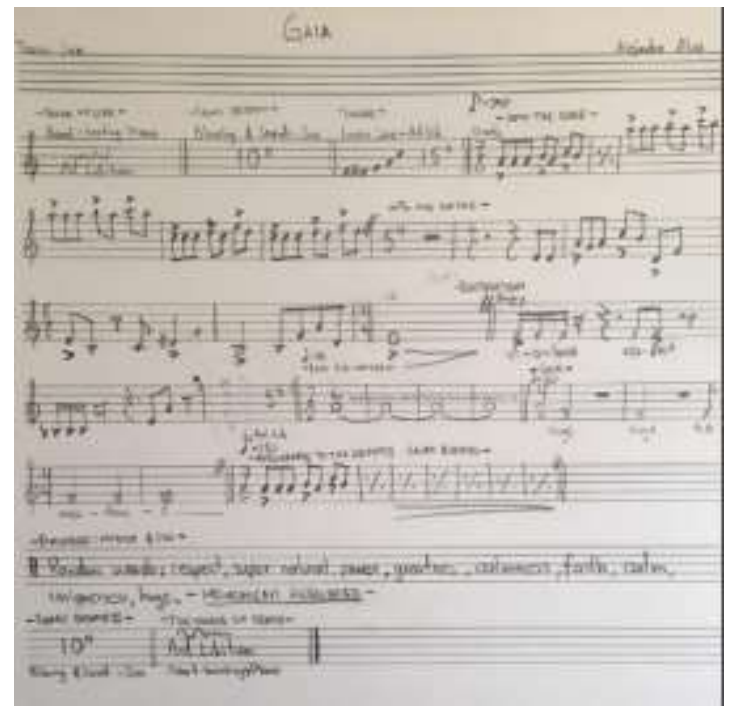




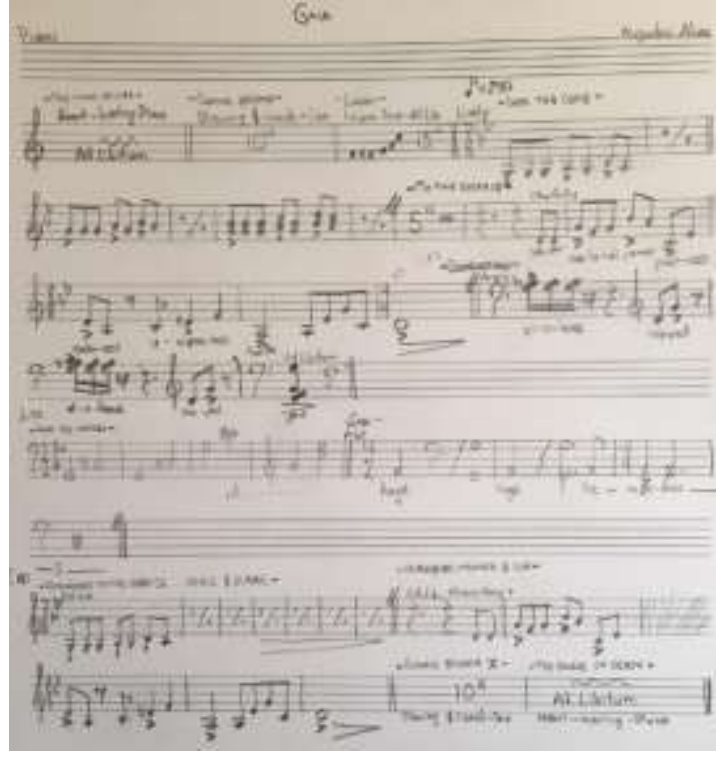

Figura 1. Partitura-guion

\section{CREATIVIDAD}

Es bien conocido por los compositores, escritores o coreógrafos que se crea desde lo que se es. ¿Qué expresa un individuo si no sus propias emociones e ideas? Al crear, el/la artista recurre a sus propios materiales afectivos $\mathrm{e}$ intelectuales para abordar un tema, elaborar un desarrollo del mismo y llegar a una síntesis. Lin Durán, coreógrafa, lo relaciona de la siguiente manera con la creación dancística:

Las derivaciones que puede tener [un] tema son infinitas y lo que el coreógrafo seleccione tendrá que ver con su experiencia de vida. Un tema es el material generador y ordenador de ideas a través de la conciencia de un coreógrafo. (1993, p. 14)

De tal manera, además de contribuir una obra artística a la sociedad, el/la artista tiene, por la naturaleza del proceso creativo, la oportunidad de evolucionar aspectos de sí mismo(a). Si bien la experiencia creativa per se es una herramienta útil que puede conducir al individuo por un camino de indagación en sí mismo y una posible restauración ulterior, la exploración de diversos lenguajes artísticos amplía aún más el posible campo de acción hacia el interior de la persona a fin de conocer qué siente y piensa, así como hacia el exterior, al usar diversos medios para expresarse e interactuar de una manera integral y coherente.

Al dirigir todo lo que somos (como organismo funcional) hacia una acción, estamos integrando nuestras potencialidades, con lo que entramos automáticamente al estado en que se facilita la creación. [...] Quedarse en el ingenio, en la emoción, en la simple energía o en la intelectualización (cada una sin las otras) implica negar la experiencia de plenitud que el arte exige. Sin plenitud, es decir, sin integración, el arte difícilmente puede surgir. (Durán, 1993, p. 45-46)

La plenitud que refiere Duran, es decir, el equilibrio entre intelecto, emoción y energía en el arte para alcanzar la plenitud, se puede extender, por analogía, a la integración entre lenguajes artísticos (e idiomas) y el subsiguiente enriquecimiento de las experiencias artística y humana. Consideremos que es natural para los creadores emprender la búsqueda de unidad de elementos variados para expresar un contenido determinado en una disciplina. De acuerdo con el compositor Reginald Smith, la composición musical es la síntesis de todas las demás disciplinas musicales, ya que reúne todas las teorías o técnicas que constituyen la esencia de la música, su interpretación y su estudio (1986, pp. 1-2).

En su ejercicio profesional como Lic. en Letras Inglesas, Lic. en Flauta Transversa, creadora multidisciplinaria y perito traductora, la autora propone la creatividad multidisciplinaria artística como un contenedor aún más amplio que puede involucrar lenguajes artísticos (e idiomas) a elección de cada persona con base en sus intereses y habilidades con el propósito de que cada quien encuentre su propia forma de unificarlos al pasarlos por el filtro de sus emociones, pensamientos, conocimientos y habilidades. En el área narrativa, Luz Aurora Pimentel plantea este proceso de la siguiente manera:

Nuestra vida está tejida de relatos: a diario narramos y nos narramos el mundo. Nuestra memoria e interés nos llevan a operar una incesante selección de incidentes a partir de nuestra vida, de la vida de los otros, del mundo que nos hemos ido narrando; una selección orientada de nuestra experiencia, para llevar a cabo una "composición" que signifique y/o resignifique esa experiencia. (2002, p. 7)

Por su parte, Carl Orff habla ya de un planteamiento multidisciplinario, de una "música elemental" necesariamente imbuida de otros elementos artísticos:

What, then, is elemental music? Never music alone, but music connected with movement, dance and speech - not to be listened to, meaningful only in active participation. (1962, p.3)

Es una realidad que, por medio del acto creativo, el/la artista puede cambiar un futuro que pareciera ineludible por medio de la indagación y la reparación de sí mismo(a). Más aún, crear usando varios lenguajes artísticos (e idiomas) a la vez permite reforzar este proceso exponencialmente. Una identidad sólida redundará en una interpretación igualmente segura. Al saber quién es y qué desea, una persona puede tomar decisiones con base en intereses y objetivos claros, lo que se traducirá, en relación con el estudio de un instrumento, en la creación de un plan de trabajo -y de vida- ordenado. Esto se reflejará en intenciones claras al organizar la vida de 
estudiante $\mathrm{y}$, posteriormente, la práctica profesional. Los individuos que tienen conciencia de sí son capaces de plantear propuestas artísticas que inviten a la sociedad que les rodea a optar por el camino del desarrollo individual y comunitario, en oposición al aislamiento y la división social. No es de extrañar que el taller pueda llevarse a cabo, además, en idioma inglés, pues el proceso de estructuración de ideas creativas artísticas permite también afianzar el conocimiento de una lengua que no es la materna.

\section{EL TALLER}

En el taller Interdisciplinary Ensembles los estudiantes crean una pieza breve que incluya música o atmósferas sonoras, uso del cuerpo (de cualquier forma) y palabras en inglés (hablando, actuando o cantando). En las primeras sesiones, la profesora comparte con los estudiantes ejercicios específicos para disparar la creatividad y mostrarles elementos que pueden usar posteriormente en sus propios proyectos: leer y analizar textos (poesía, narrativa, drama); ver y analizar videos (de danza, música, teatro, clases magistrales, etc.); trabajo de la conciencia corporal (cómo pararse, cómo caminar, espacialización en el escenario, conciencia de que el cuerpo expresa en todo momento). En el área musical, los participantes tienen total libertad creativa, pues es su área de especialidad. Al respecto, la profesora sólo hace sugerencias para aclarar ideas y cuestionamientos para afianzar la conciencia sobre el proceso de creación. Se hace especial énfasis en el hecho de que los estudiantes son músicos, instrumentistas, que experimentan el mundo como tales y deben proyectar esa visión en sus piezas. Por lo tanto, deben estar conscientes de las posibles relaciones entre música y otros lenguajes artísticos al crear una pieza propia con elementos de diversas disciplinas, considerando que la música es un contenedor, el lenguaje básico a partir del cual se pueden derivar analogías. En relación con los paralelismos entre lenguajes artísticos desde un punto de vista narrativo, Pimentel refiere:

La narratividad trasciende no sólo fronteras genéricas y modales sino semióticas, puesto que lo narrativo puede observarse en diferentes medios y sistemas de significación. Incluso este fenómeno transemiótico ha propiciado la extensión conceptual, no sólo de lo narrativo, sino del término lenguaje, mismo que se aplica ahora ya no exclusivamente al verbal sino a otros sistemas de significación y de representación. (2002, p. 13)

Las primeras clases con ejercicios específicos, planeadas para detonar la creatividad, se dividen en dos partes: 1) Conciencia corporal: se exploran sensaciones, el uso del cuerpo como intérprete y la conciencia del propio cuerpo en el escenario; 2) Análisis de textos literarios originalmente escritos en inglés y su representación sonora/corporal/escénica. Se lee y analiza un texto con características específicas a fin de dar a conocer a los alumnos ejemplos de usos del idioma inglés que pueden usar en sus propios proyectos. En todo momento, la profesora hace señalamientos en relación con la profesión del intérprete con base en analogías con los ejercicios en curso y en función de las reacciones de los alumnos (lectura de los textos musicales, interpretación en el propio instrumento, conciencia escénica). Es importante destacar que se busca que los estudiantes no sólo practiquen el inglés, sino que conozcan y exploren su dimensión literaria, en analogía con otros lenguajes artísticos, especialmente el musical, por ser su área de especialización. Es por ello que en todo momento se hacen paralelismos entre el análisis literario, musical y corporal. Se presentan aquí dos ejercicios específicos representativos del trabajo con textos en inglés.

\section{EJERCICIO ESPECÍFICO INTRODUCTORIO}

El primer ejercicio específico, introductorio al taller, es el análisis de "Sound and Sense", fragmento de An Essay on Criticism de Alexander Pope. Puesto que los alumnos son músicos, el taller está enfocado al uso de otros lenguajes artísticos desde la perspectiva musical. La práctica del inglés, por lo tanto, se concentra en su uso literario, presentando posibles analogías entre el lenguaje literario y el lenguaje musical. Tras revisar el posible vocabulario problemático para cerciorarse de que los alumnos comprenden cabalmente el texto, en este ejercicio se analizan el ritmo del poema (stress, foot, meter, iambic pentameter, rhyme, heroic couplet); la cohesión fonológica (repetición de sonidos) y las imágenes poéticas sonoras, recursos por medio de los cuales el autor ejemplifica que "the sound must seem an echo to the sense". A partir del análisis del texto, la profesora guía a los asistentes a fin de explorar el ritmo físicamente, haciendo movimientos que ayuden a experimentar, durante la lectura en voz alta, el fraseo musical con base en la pronunciación correcta de las palabras y la estructura métrica pues, como señalan Traugott y Pratt: "a given meter can be described as a system of potentials for stress, somewhat like a musical beat." (p. 75) Después de afianzar la estructura rítmica básica, se crean con la voz y el cuerpo atmósferas sonoras que representen las imágenes poéticas, enriquecidas con frases corporales. En esta parte del proceso se trabaja también la composición escénica con base en los movimientos que van diseñando los participantes. Conforme los participantes se relajan y participan corporalmente, la profesora los invita a reflexionar qué tan conscientes están como instrumentistas de cómo usan el cuerpo al tocar. En sesiones subsiguientes se profundiza este trabajo de reflexión pues al proponer sus propias piezas para ser interpretadas junto con sus compañeros, es necesario que cada quien comparta con los demás qué pueden hacer técnicamente con su instrumento. En este punto, la profesora aprovecha para pedir a cada quien que también comparta un poco de la técnica básica de su instrumento, como si fueran profesores de los demás participantes, prestando especial atención a cómo se usa el cuerpo para tocarlo, pues los demás 
asistentes tocan otros instrumentos. De nuevo a partir de preguntas y pidiendo a los participantes que ejemplifiquen, la profesora conduce la exploración de la conciencia corporal en cada instrumento, invitando a los compañeros a plantear sus dudas como si ellos mismos fueran a estudiarlo.

Además de que prestar especial atención a la sonoridad del idioma en inglés es inherente en el trabajo con estudiantes de las diferentes licenciaturas en música, es necesario resaltar la importancia de generar conciencia sobre las diferencias entre la palabra escrita y hablada pues, de acuerdo con Traugott y Pratt:

One of the greatest difficulties many people have in thinking about the sounds of a language is in separating sounds from spelling. It is part of the high cost of literacy that many of us cease to hear ourselves and others accurately; instead we rely heavily on the visual image we have of words in their written form. (1980, p. 42)

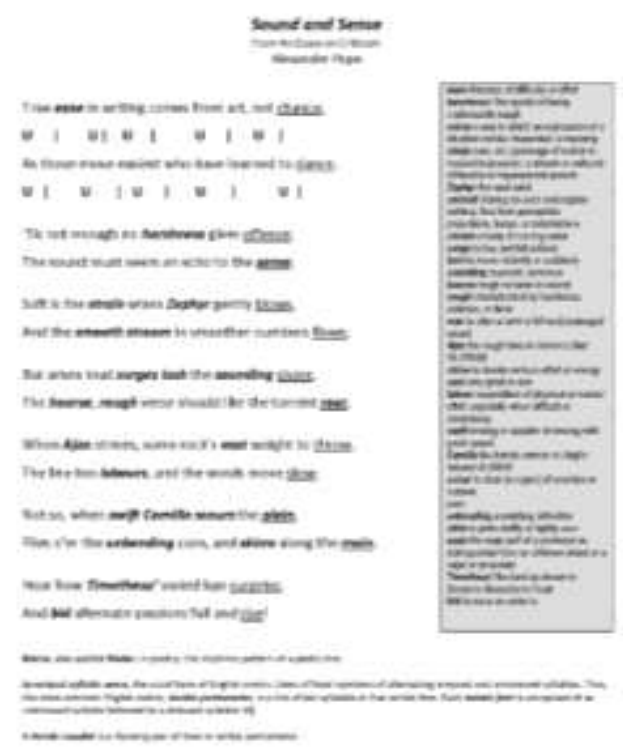

Figura 2. Ejercicio específico introductorio

\section{EJERCICIO ESPECÍFICO DE DRAMATIZACIÓN}

Se realiza la lectura dramatizada de alguna escena de Alice's Adventures in Wonderland, de Lewis Carroll. Ya que se trabaja especialmente con instrumentistas, se exploran la expresividad y el fraseo con base en el análisis del texto (narrador, personajes, diálogo) en analogía con el discurso musical, así como la presencia y la conciencia escénicas, aun al tratarse de una dramatización leída pues, en sus recitales, los alumnos suelen leer la música que interpretan y se pretende que apliquen lo explorado en su práctica musical cotidiana. Dada la naturaleza del texto, la adaptación escénica del mismo se presta para un histrionismo exacerbado que permite un montaje lúdico mientras se practica, además, la pronunciación del idioma inglés hasta lograr fluidez en los diálogos y/o la narración.

En el taller se da diferente tratamiento sonoro/escénico a los diversos géneros literarios que eligen los estudiantes para sus proyectos, pues cada uno tiene diferentes características y, por lo tanto, al trasladarlos a la escena requieren diferentes recursos. Los estudiantes exploran los textos como intérpretes, sin importar el género elegido, relación que, de acuerdo con Traugott y Pratt, sucede naturalmente cuando un intérprete trabaja un texto dramático:

In terms of the gap between speech and writing, drama occupies an interesting position between oral and written literature. As a rule, dramas in the modern period are composed in writing and intended to be executed orally. [...] The text of a drama consists primarily of represented speech [...] Ultimately, the gap between speech and writing in drama is filled by the performers themselves, and this is one of the main reasons why no two productions of a play will ever be alike. Part of the richness of drama as a genre lies precisely in its openness to interpretation in this regard. (1980, pp. 44-45)

Conforme los estudiantes comienzan a idear sus proyectos personales, las sesiones se van enfocando en concretarlos con la colaboración de todos los participantes. La exploración y la retroalimentación colectivas son cruciales para consolidar gradualmente la confianza de los estudiantes durante el proceso creativo, de montaje e interpretación. Posteriormente, la profesora los guía y acompaña proporcionándoles los recursos que considera adecuados para cada proyecto, de conformidad con las necesidades y los intereses específicos de cada participante. Se logra coherencia entre los diversos elementos de los lenguajes utilizados principalmente por medio de la analogía. Por ejemplo, si cambiamos la palabra "coreográfico" por "musical" o "literario" en el siguiente párrafo de Lin Durán, podemos encontrar que los mismos principios aplican a diversas disciplinas artísticas, a fin de usarlos al crear una pieza multidisciplinaria enfocada en la música:

El lenguaje coreográfico "habla", en primera instancia, a los sentidos, especialmente a la percepción visual, auditiva (la música) y táctil (cinética) del espectador. Estos estímulos se conectan con nuestras vivencias más significativas, aquellas que hemos guardado como experiencias tristes y alegres, o simplemente impactantes. A esta conexión se empalma un proceso de pensamientos estético (no analítico), basado en unidad, claridad, contraste, conflicto y coherencia. Estas cualidades, entre otras, se muestran continuamente en la naturaleza y quedan grabadas en nuestros hábitos de percepción, por lo que el espectador siente la necesidad de ponerse en armonía con ellas. (1993, p. 14)

Puesto que el taller está dirigido principalmente a 
instrumentistas, el objetivo más importante es lograr que los estudiantes se sientan cómodos y con confianza al crear e interpretar sus propias piezas. Sobre todo, el taller se trata de atreverse y explorar la creatividad, experimentar, más que de alcanzar un resultado específico. Al igual que en el método planteado por Maria Montessori: "the lesson corresponds to an experiment." (p. 123) Es crucial que los estudiantes, quienes están acostumbrados a entrenarse para tocar música compuesta por otros, exploren sus emociones y sentimientos a fin de descubrir qué desean expresar con su propia voz. Además,

Student performers can benefit particularly from composition. They are often the most reluctant to become involved in it, since they need to spend so much time in instrumental practice, but they will never play music really superbly, in spite of all their technique, without inside experience; with it, they will be able to recreate the music just as the composer himself conceived it. (Smith, 1986, p. 2)

Esto refuerza también el enfoque multidisciplinario personal de la profesora: en este taller, se busca la experiencia de las diferentes disciplinas desde el interior de las mismas, dando el debido lugar a cada una de ellas, conociendo sus fundamentos, respetando la complejidad de cada una y no solamente reuniendo elementos diversos que den como resultado un catálogo de recursos, obras sin coherencia interna. En este taller, la exploración no se basa en el conocimiento teórico específico de ningún área involucrada (el cual, no obstante, deben poseer los estudiantes), sino en las intenciones expresivas de los estudiantes y en el acto mismo de encontrar relaciones entre elementos aparentemente disímiles. Posteriormente, el conocimiento específico servirá para concretar las ideas planteadas. Como menciona Durán: "el artista se conduce en su trabajo creativo a través de impulsos sensibles y por intuición (acumulación de experiencias)". (p. 14) Por lo tanto, se busca que los estudiantes usen lo que ya saben de manera espontánea, guiados por sus emociones, gustos e ideas personales. En todo momento, la profesora los insta a volcar todo lo que son en sus proyectos, a fin de crear piezas llenas de contenido humano y coherencia artística.

\section{EJERCICIO ESPECÍFICO DE CREATIVIDAD ASISTIDA CON EL DICCIONARIO}

Los estudiantes cortan una hoja tamaño carta en ocho partes iguales. En sus celulares, conectándose a la red de Internet de la FaM-UNAM, abren el diccionario Merriam-Webster, escriben en un papel la palabra del día y luego hacen clic en la misma. Leen las definiciones y, de entre ellas, eligen la palabra que más les llame la atención, sea por su significado o sonoridad, luego la escriben en otro papel. Hacen esto sucesivamente hasta juntar ocho palabras. En el transcurso, en caso necesario, los participantes aclaran sus dudas sobre la pronunciación y el significado de las palabras elegidas por medio del mismo diccionario.

Con base en el ejercicio introductorio sobre los acentos del texto, los estudiantes escriben debajo de cada palabra su ritmo correspondiente usando П para sílabas átonas y | para sílabas tónicas) (e.g.: musical, $\mid \sqcap$ П). Una vez que los asistentes tienen sus ocho palabras escritas en los pedazos de papel, con todo y su ritmo correspondiente, conocen su pronunciación y su significado claramente, deben ordenarlas para crear una secuencia lógica. Los criterios de orden quedan a su libre elección.

Las secuencias así generadas se trabajan entonces en dos vertientes: a) con base en su contenido; b) con base en su sonoridad. Los participantes comparten sus secuencias en voz alta, uno a uno. Comentan sus criterios personales de selección de palabras, comparten verbalmente el contenido que desarrollaron a partir de la ordenación de las mismas y lo plasman por escrito. Se presta especial atención al orden lógico del discurso, en paralelismo con el discurso musical. A este respecto, Luz Aurora Pimentel refiere la gran importancia que tiene la secuencia para plantear una postura personal por medio de la creación narrativa:

La velocidad a la que se narra, la secuencia elegida, la cantidad de detalles con los que se describe un objeto, su composición, la perspectiva que se elige para narrar [...] las estructuras narrativas en sí son ya una forma de marcar posiciones ideológicas. (2002, p. 9)

Una vez que los asistentes tienen claro el contenido de su secuencia, se concentran en la interpretación de la misma desde una perspectiva musical, basándose en la línea melódica y el ritmo naturales del habla, concentrándose en transmitir como ejecutantes el contenido que ellos mismos desarrollaron previamente. Finalmente, las ideas musicales se representan, por analogía, con el cuerpo, tomando en cuenta acentos, fraseo, espacialización, capacidades y habilidades físicas de los participantes. Como se mencionó anteriormente, Orff planteó en su enfoque que es inherente al ser humano la integración de música, movimiento y palabra (en su caso, especialmente, cantada).

That the unity of music and movement is still natural present in the child (adolescents have already lost it, and must relearn it) is so sadly overlooked that it became the cornerstone of my new pedagogical work. I suddenly understood what the first Shulwerk had lacked: the singing voice, the word. A child quite naturally starts with a call, a rhyme, with text and tune together; movement play and song coalesce and integrate. (1962, p. 3)

Los asistentes pueden mostrar individualmente sus interpretaciones o, mejor aún y si el tiempo lo permite, hacer 
que sus compañeros participen en su ejercicio creativo, dándoles instrucciones tomando en cuenta el trabajo de ensamble. La profesora acompaña señalando paralelismos con la práctica musical. Por ejemplo: como en un ensayo de música de cámara en el que los integrantes se deben poner de acuerdo en relación con los criterios interpretativos de las obras montadas; estar atentos y sensibles a lo que hacen los demás a fin de lograr una verdadera integración de todos los miembros del ensamble; así como saber seguir instrucciones de un director o compositor, según sea el caso, como instrumentistas.

Conforme los estudiantes comparten sus ejercicios, la profesora comenta si existe congruencia entre el contenido del texto y su interpretación musical (e.g.: un tempo lento para un texto que hace referencia a la lentitud; la relación entre los giros melódicos y los acentos tanto de las palabras como del fraseo); así como entre el texto, su representación musical y corporal (e.g.: fraseos musicales y corporales, carácter del lenguaje corporal) e invita a hacer las adecuaciones correspondientes, en su caso, para lograr coherencia entre la interpretación y el texto desde las perspectivas musical y escénica:

Organización funcional coherente de los elementos del todo. Esto implica que la construcción se base en contrarios que den la tensión dinámica (conflicto) y elementos contrastantes, que den ritmo a la obra y subrayen los contenidos importantes. (Durán, 1993, p. 15)

En cuanto al manejo del cuerpo, la profesora señala en el momento cómo conforme los estudiantes asimilan el sentido musical del texto, integran naturalmente su propio lenguaje corporal para expresarse, por lo que es importante que antes de interpretar en su instrumento comprendan cabalmente el texto musical en todos sus aspectos y permitan también que su cuerpo interprete musicalmente lo que ya comprendieron intelectualmente, pues esto les permitirá tocar incluso sin o con muy poca tensión, en la medida en que tengan claridad de lo que desean "decir" con el discurso musical.

El objetivo principal en este ejercicio es demostrar a los alumnos que se puede aprovechar cualquier elemento externo para detonar la creatividad, que la mayor parte de los recursos usados para crear proviene del interior de cada persona, que cada quien comunica en sus creaciones lo que siente, lo que piensa, sus experiencias personales. En relación con la danza, Lin Durán comenta que:

El material temático, por muy platónico o metafísico que sea, tiene su marco de referencia en lo humano. Mientras más humano, más profundo es el impacto que el público recibe, ya que el espectador tiende a identificarse con los contenidos que se filtran por el tejido coreográfico. (Durán, 1993, p. 13)
La profesora invita a los alumnos a observar que, aun partiendo de la misma palabra, cada quien generó un resultado creativo diferenciado debido a sus características personales. Los asistentes discuten y analizan el proceso y el estilo de cada quien, en el entendido de que:

Estilo es el sello personal que el artista imprime a su obra. [...] El estilo es lo peculiar y lo diferencial que identifica grupos de obras. (Durán, 1993, p. 31)

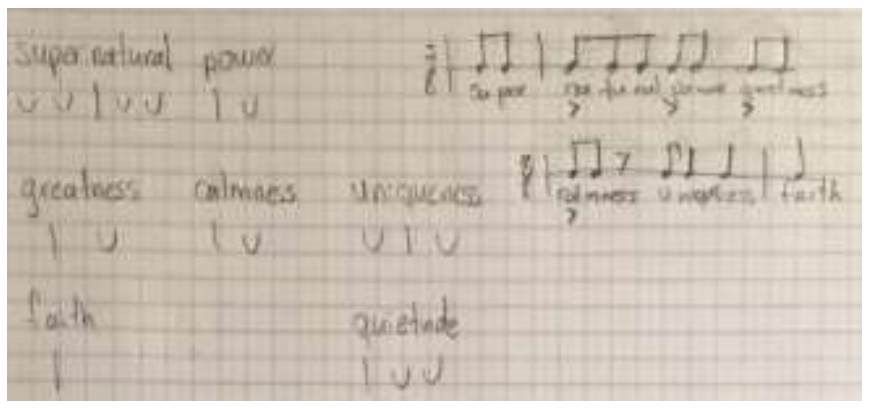

Figura 3. Ejercicio específico de creatividad asistida con el diccionario

En cuanto a la práctica del idioma, por medio de este ejercicio, en un primer nivel, los estudiantes adquieren vocabulario nuevo, practican y afianzan su pronunciación y significado gracias al uso integral del mismo en tanto que resignifican las palabras de acuerdo con sus intereses, pensamientos y emociones. En un segundo nivel, los estudiantes exploran el uso expresivo del inglés al desarrollar contenido creativamente en relación con una secuencia de palabras sueltas. En un tercer nivel, los participantes hacen un paralelismo entre el lenguaje verbal y la práctica musical, al igual que en el ejercicio introductorio, pero con el valor agregado de que en esta ocasión trabajan con sus contenidos intelectuales, afectivos y estéticos personales.

\section{EJERCICIO ESPECÍFICO DE ANÁLISIS DE VIDEOS}

Se analizan tres videos relacionados con el trabajo corporal y dancístico: 1) "Rehearsing The Royal Ballet's Sleeping Beauty"; 2) fragmentos de una clase magistral de Maxim Vengerov; y 3) "A Choreographer's Creative Process in Real Time".

En el primer video se revisa el proceso de montaje de un solo actuado en el ballet Sleeping Beauty:

A dancer new to the role of Carabosse works with Monica Mason on Carabosse's arrival at Princess Aurora's christening. The rehearsal focuses on expressing character and storytelling through gesture. (Royal Opera House)

A través de este video, se analizan los elementos de interpretación corporal de un personaje. La profesora deriva y comenta analogías directas con la interpretación de un texto musical en un instrumento. Se discuten en grupo los efectos 
dramáticos y escénicos que pueden tener en el público los detalles corporales trabajados durante el ensayo. También, se comenta la coherencia entre el lenguaje corporal, la música y la historia contada, considerando la referencia de Lin Durán:

La coherencia tiene que ver con los procesos orgánicos de la estructura. Tiene que ver con que los elementos se relacionen entre sí con lógica perceptual y racional, con unidad. Sentimos y sabemos que las relaciones tienen sentido, que "nada sobra ni falta" y que el tejido no es disparatado ni está lleno de agujeros, sino que tiene consistencia. (1993, p. 30)

También, se aborda el concepto de coherencia desde la experiencia que ya tienen los alumnos tocando el repertorio correspondiente a su instrumento y en ensambles. Igualmente, se busca integrar los conocimientos que ya tienen de análisis musical para analizar estructuras en otros lenguajes artísticos, siempre con base en los conceptos generales de analogía y contraste.

La profesora señala la importancia de las imágenes mentales para interpretar con el cuerpo, la conciencia en relación con el contexto (historia) y las características del personaje para expresar ideas claras tanto física como musicalmente; que el ensayo deja ver que nada se deja al azar, cada gesto se planea, incluso el número de pasos se practica hasta asimilarlo intelectual, emocional y corporalmente. Se propicia la discusión de la actuación sin palabras, de la traducción de la música al lenguaje corporal sin pasar por la palabra hablada, así como las diferencias entre la interpretación de la joven y de Mason, bailarina retirada en quien se perciben experiencia y madurez técnicas, expresivas, intelectuales, estéticas y emocionales en comparación con las experiencias de los propios estudiantes y lo que perciben en músicos de gran trayectoria, incluyendo a sus maestros. Se comenta el efecto de la técnica y el trabajo detallado de montaje de obras en las diversas disciplinas en relación, en todo momento, con el quehacer musical cotidiano de los participantes tanto durante el estudio como en el trabajo de ensamble y las presentaciones escénicas.

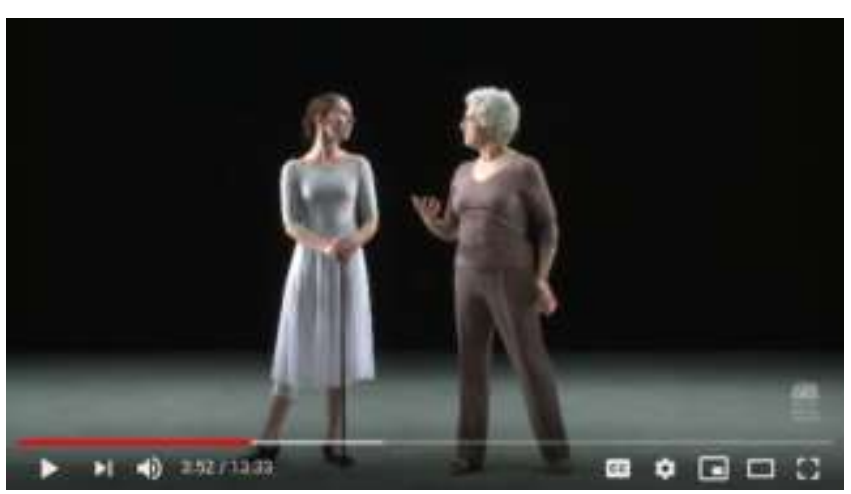

Figura 4.1. Ejercicio específico de análisis de videos.

En el segundo video, "Maxim Vengerov - Masterclass", se analiza el uso de imágenes visuales e historias para aumentar la expresividad interpretativa. Comenta el violinista:

I use these images to explain the students what to do with music. But I'm just trying to give them a chance to think about this music themselves, to create something that music will be so alive and so much vivid because the imagination is part of our human existence. (Vengerov)

Se hace énfasis en el uso de la imaginación en la interpretación musical, así como la invitación a llevarla aún más allá, creando. Continúa Vengerov:

Whatever you do, you can't actually go wrong if you take the music first and according to that you create an atmosphere, even the dialogues, even question and answer, this is so interesting to explore...

Este video sirve para explorar la analogía entre las imágenes visuales y la música. En el video se parte de la música, pero el interés de presentar este material es que los estudiantes comprendan que se puede partir de cualquier lenguaje artístico para crear analogías y un discurso multidisciplinario en donde todos los elementos estén amalgamados entre sí.

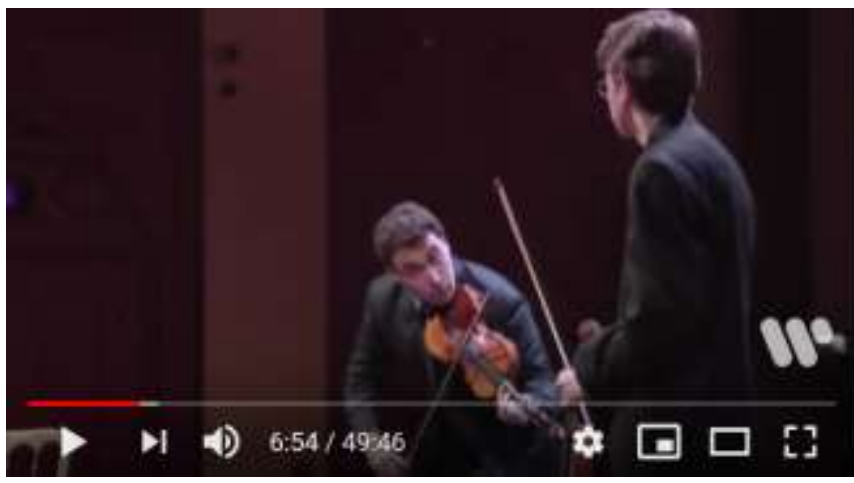

Figura 4.2. Ejercicio específico de análisis de videos.

En el tercer video, en contraste con el ejercicio de interpretación, se revisa el proceso coreográfico creativo desde la perspectiva de Wayne McGregor, coreógrafo, donde:

Creativity for me is something that is absolutely critical [...] it's something that you can teach, the technicities of creativity can be taught and shared, and I think that you can find things about your own personal physical signature, your own cognitive habits and use them as a point of departure to misbehave beautifully.

Tras varias sesiones de ejercicios específicos para disparar los procesos creativos, este es el primer ejemplo de un creador en acción que se propone a los estudiantes. Esto es importante para los fines del taller, pues los asistentes son, generalmente, estudiantes instrumentistas, quienes adquieren el entrenamiento para interpretar obras de otros. Se presenta el 
testimonio del creador dancístico a fin de que los participantes escuchen de viva voz lo que piensa y siente un creador en torno al proceso creativo. En las primeras sesiones del taller, por medio de los ejercicios específicos, la profesora resalta la importancia que tiene para los intérpretes experimentar la creatividad a fin de explorar los diversos significados que se pueden imprimir a las obras posteriormente interpretadas. McGregor da testimonio del descubrimiento de la coreografía para expresarse como individuo:

That was the first time that I found an opportunity to feel that I was able to express my own voice and that's what has fueled me then to become a choreographer, I felt like I have something to say and something to share.

Se invita a los alumnos a que compartan sus experiencias en torno al proceso creativo: si han creado o no; si se sienten ajenos a los procesos creativos o parte de los mismos (generalmente, los estudiantes instrumentistas consideran que no tienen herramientas o incluso derecho a componer pues no estudian composición); si han imaginado que pueden crear algo, entre otros. Para esta sesión, los asistentes ya deben estar generando ideas de sus propios proyectos.

Una vez más, la profesora señala la importancia de la analogía entre elementos de diversas disciplinas, explica cómo el coreógrafo deriva la traducción de estímulos externos al lenguaje corporal e invita a los alumnos a discutir analogías en general:

I'm exploring the space of " $t$ " and flashing through it with some action. [...] So, we're starting to build a phrase. [...] So, I have taken this aspect of TED and translated it into something physical. [...] Think about that word "TED" in front of you [...] what I'd like you to do is transplant that outside into the real world. (McGregor)

En relación con el idioma inglés, en este ejercicio los estudiantes practican la escucha de diversos acentos $\mathrm{y}$ adquieren vocabulario relacionado con el uso del cuerpo y el escenario que les será útil al dar instrucciones a sus propios compañeros en sesiones posteriores al realizar los montajes de sus propias creaciones, así como en clases magistrales y en la vida profesional.

Se discuten, también, los tres métodos que usa McGregor a lo largo del video para improvisar creativamente:

The first [method]: body to body transfer with an outside mental architecture that I work, they hold memory for me; the second [method] which is using them [dancers] as objects to think with their architectural objects, I do a series of provocations: "if this happens, then... if that happens" [...]; and this is the third method, [...] a task-based method where they [the dancers] have the autonomy to make all of the decisions by themselves [...] we're gonna' do a mental dance.

La profesora señala, para concluir con los ejercicios específicos y pasar en sesiones subsiguientes al trabajo de los proyectos personales de los estudiantes, que cada uno de ellos tiene total libertad de elegir sus métodos creativos (lo que previamente se consideró como pretextos externos para detonar el proceso creativo), así como el contenido de sus creaciones. El objetivo de los ejercicios específicos fue tan sólo mostrarles posibilidades de recursos y formas de usarlos en sus propios trabajos, en las siguientes sesiones el trabajo se concentrará en el desarrollo de sus propias ideas.

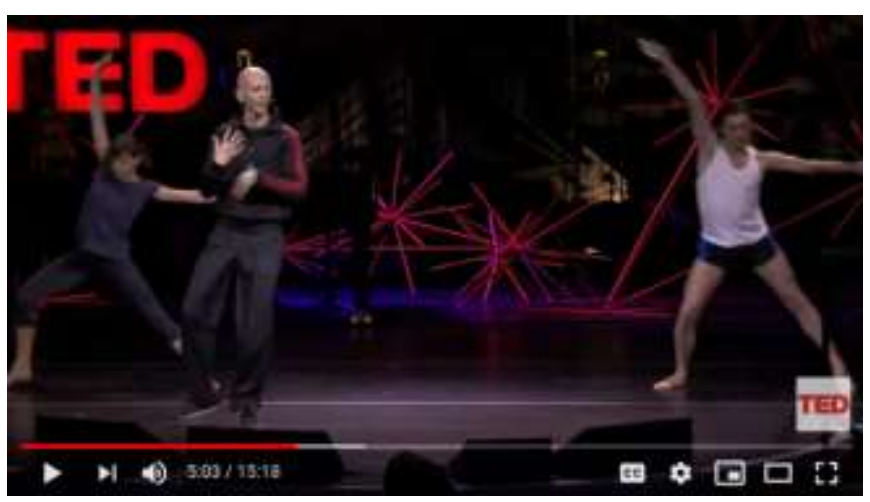

Figura 4.3. Ejercicio específico de análisis de videos.

\section{EXPERIENCIA OBTENIDA Y FUTURO DEL TALLER}

Al plantearse redactar diversos trabajos escritos sobre lo realizado en el taller, la autora ha llevado a cabo un minucioso análisis de las suposiciones previas a su creación; la planeación de cada uno de los ejercicios planteados en el mismo; los registros de observación de experiencias con los estudiantes; así como sus propios comentarios a las experiencias continuas a manera de diario de trabajo. Este proceso le ha conducido a descartar ejercicios que no han proporcionado experiencias sustanciales a los estudiantes; continuar analizando y desarrollando aquellos que sí lo hacen; así como diseñar ejercicios que pondrá a prueba en semestres venideros.

Empero, un obstáculo para el desarrollo óptimo del taller es la poca concurrencia debido a que muchos estudiantes desconocen la existencia del mismo; al temor de crear que sienten algunos instrumentistas que llegan a la primera sesión $\mathrm{y}$ descubren que las actividades giran en torno a su propia creatividad; y que no todos los estudiantes que saben de la existencia del taller consideran importante la formación artística multidisciplinaria. Con más alumnos, se podría llevar a cabo una mayor interacción entre participantes, lo que redundaría en beneficio de su práctica del idioma y el proceso creativo. También, la autora podría probar con mayor diversidad de personas los ejercicios planteados y, en su caso, 
realizar modificaciones para satisfacer las necesidades de perfiles diferentes a los recibidos hasta el momento. Además, enfrentar otras combinaciones de perfiles dentro de los grupos permitiría una mayor exploración de recursos artísticos por parte de la autora con los estudiantes y, a la vez, de los participantes en sus propias obras.

Gracias al espacio de experimentación creado con la aprobación de la FaM-UNAM, la autora no sólo ha logrado ofrecer a los estudiantes un espacio de creatividad artística y afianzamiento de los conocimientos musicales y de inglés adquiridos en otras materias, sino que desarrolla una metodología de trabajo de integración multidisciplinaria creativa que se ha probado también con estudiantes de danza del profesor José G. Ornelas Rodríguez; con sus propios estudiantes de flauta de la Escuela de Iniciación Artística núm. 1 del INBAL; $\mathrm{y}$, recién, con profesores de otras instituciones como son el CIMI de la Universidad Veracruzana (Samadhi Méndez Suárez, flautista) y la ENES Morelia (Pedro Salvador Velasco, percusionista) (esta última, iniciativa propia de la autora, de manera independiente y gratuita, con profesores voluntarios de las instituciones mencionadas). Esto constituye la aplicación y difusión de lo explorado en el taller a manera de investigación-acción con el interés de beneficiar a la comunidad musical y de otras áreas artísticas.

Es importante también mencionar que una limitante de este taller específico para que la autora comparta con más docentes las experiencias de manera que éstos las puedan replicar en otros contextos es que el mismo fue planteado desde la formación profesional, la experiencia y los intereses multidisciplinarios de la autora. Por ello, continúa explorando las diversas ramificaciones de las experiencias desarrolladas en el taller en versiones en español, dirigidas al trabajo con estudiantes y profesionales de otras disciplinas artísticas como son la danza y la literatura; así como con público en general, a manera de sensibilización artística. Por ejemplo, en 2018 la autora escribió un artículo aprobado por ABRAF (Associação Brasileira de Flautistas) con enfoque específico en los beneficios del taller en términos de enriquecimiento de la práctica interpretativa en flautistas, así como en el sentido del estudio para los estudiantes de este instrumento. De igual manera, en 2019 presentó un cartel enfocado en la relación entre la práctica musical y la familia en una Jornada Académica FaM-University of Sheffield dirigida especialmente a profesionales de la psicología. El presente trabajo concentrado en la práctica del idioma inglés es otro ejemplo.

La autora considera que los trabajos breves y específicos como los antes mencionados abonarán a su proyecto a largo plazo de publicar un trabajo de mayor envergadura en el que se presenten modelos de clase comentados, en los que se describan ejercicios modelo específicos para sensibilizar a los participantes en diferentes disciplinas artísticas; los procesos de decisión de la autora para plantearlos tal como se muestran a partir de su formación y experiencia profesional y como docente; las observaciones basadas en la práctica con estudiantes de diversas áreas; así como investigaciones teóricas relevantes. Es evidente, por tanto, que se tiene por delante un largo camino de experimentación con estudiantes y docentes de artes (principalmente) interesados por integrar la práctica artística multidisciplinaria creativa en los procesos formativos y la práctica profesional de unos y otros. Por medio del trabajo colaborativo con docentes a partir de situaciones específicas a resolver, la autora podrá trabajar en proponer líneas de trabajo con base en las cuales ellos, a su vez, le proporcionarán retroalimentación que servirá de mirada y opinión externas que permitirán enriquecer gradualmente los futuros materiales que servirán de referencia para estudiantes y profesores por igual.

\section{REFERENCIAS}

[1] Biget, Arlette. (1998). Une pratique de la pédagogie de groupe dans l'enseignement instrumental. Cité de la musique, Département pédagogie et documentation musicales.

[2] Cobo Dorado, Karina. (2015). La pédagogie de groupe dans les cours d'instruments de musique. Siciences de l'éducation musicale, L'Harmattan.

[3] Crousier, Claude. (2001). Le musicien et le groupe, le point de vue d'un professeur de clarinete. Cité de la musique, Département pédagogie et documentation musicales.

[4] Durán, Lin. (1993). Manual del Coreógrafo. INBA, CENIDI-Danza.

[5] Garibaldi Pérez Vilchis, Thelma Jaet. Programa de asignatura Interdisciplinary Ensembles I. (2017). Universidad Nacional Autónoma de México.

[6] Montessori, Maria, Ed. Gerald Lee Gutek. (2004). The Montessori Method, The Origins of an Educational Innovation: Including an Abridged and Annotated Edition of Maria Montessori's The Montessori Method, Rowman \& Littlefield Publishers, Inc.

[7] Orff, Carl. (1963). April-May. Music Educators Journal. "The Shulwerk Approach" (speech given at the opening session of the Orff elementary education course, University of Toronto, July 23August 3, 1962).

[8] Pimentel, Luz Aurora. (2002). El relato en perspectiva. Siglo XXI Editores.

[9] Pope, Alexander. From Essay on Criticism. Oxford University Press. (1973). The Oxford Anthology of English Literature (Vol. 1).

[10] Royal Opera House. Rehearsing The Royal Ballet's Sleeping Beauty. [video]. https://www.youtube.com/watch?v=Q74fEYxMsuk

YouTube.

[11] Smith, Brindle Reginald. (1986). Musical Composition. Oxford University Press.

[12] Ted Talks. A Choreographer's Creative Process in Real Time | Wayne McGregor | TED Talks. [video]. YouTube. https://www.youtube.com/watch?v=KPPXXeoIzRY

[13] Warner Classics. Maxim Vengerov - Masterclass. [video]. YouTube. https://www.youtube.com/watch?v=Wpp7oxrBUq0 